\title{
The Adaptive Random Access Carrier Allocation Scheme in NB-IoT Networks
}

\author{
Yen-Wen Chen, Guan-Yi Xue \\ Department Name of Communication Engineering, National Central University, Tao-Yuan \\ Email:ywchen@ce.ncu.edu.tw
}

How to cite this paper: Chen, Y.-W. and Xue, G.-Y. (2022) The Adaptive Random Access Carrier Allocation Scheme in NB-IoT Networks. Communications and Network, 14, 1-11.

https://doi.org/10.4236/cn.2022.141001

Received: November 1, 2021

Accepted: December 11, 2021

Published: December 14, 2021

Copyright $\odot 2022$ by author(s) and Scientific Research Publishing Inc. This work is licensed under the Creative Commons Attribution International License (CC BY 4.0).

http://creativecommons.org/licenses/by/4.0/

(c) (i) Open Access

\begin{abstract}
The rapid progress of the deployment of IoT services pushes the evolution of wireless communication techniques. Because the number of IoT devices is much more than that of the human-held devices for traditional services. It introduces the random access issue in radio networks. In order to support massive IoT devices to transmit data in NB-IoT, the release 14 of 3 GPP provides the preambles in non-anchor carrier for random access. However, if more non-anchor carriers are provided for random access, the resource of uplink shared channel will be compressed. The use of non-anchor carrier for random access preambles shall be carefully allocated for effective resource utilization. In this paper, we propose the adaptive non-anchor allocation algorithm by referring to the collision report flag (CRF) from the user equipment. The proposed CRF algorithm considers the congestion status of uplink to adjust the number of non-anchor carriers in flexible way for better random access experience of huge random access attempts condition. The simulation results show that the proposed algorithm achieves high success access ratio and effective non-anchor carrier utilization when comparing to that of the fixed allocation schemes. The proposed scheme can save 5 - 10 numbers of non-anchor carriers for the number of UEs varies from 15,000 to 37,500 when comparing to the fixed 15 non-anchor carriers scheme under the similar successful access ratio.
\end{abstract}

\section{Keywords}

NB-IoT, Random Access, Preamble, Radio Resource Allocation

\section{Introduction}

Recently, the concept of internet of things (IoT) is widely applied in many fields, such as environment monitoring, industry automation, safe monitoring and 
control, disaster prevention, etc. Comparing to traditional human-based communication services, the IoT services need to accommodate much more end devices though the required bandwidth is much less than the traditional services. The main characteristics of the IoT services are high frequency network access with short connected time. In order to support the frequent network access from huge number of IoT devices for data transmission, the uplink radio resource shall be properly allocated so that the detected data can be transmitted under the delay constraint.

Third Generation Partnership Project (3 GPP) proposed the preambles in long term evolution (LTE) network to deal with the contention of random access in $4 \mathrm{G}$ communication system. The user equipment (UE) shall choose one preamble to inform the evolved Node B (eNB) for the need of uplink resource before transmission. If more than one UE happened to choose the same preamble, then the collision will occur and the access will fail. Although the release 13 of 3 GPP improves the scheduling for uplink from original LTE which determines the length of Narrowband Physical Uplink Shared Channel (NPUSCH) by the number of subcarriers and slots in narrow band IoT (NB-IoT), the access congestion of massive end devices is not eased due to the fixed anchor carrier [1]. In release 14, $3 \mathrm{GPP}$ proposes the flexible adoption of non-anchor carriers to support random access preambles, it is helpful to relax the congestion situation [2] [3]. However, the more non-anchor carriers used for access preambles the less bandwidth can be supported for data transmission in NPUSCH. Hence we shall allocate the non-anchor carriers by considering the congestion status in proper way. This paper proposes the adaptive non-anchor carrier allocation algorithm to flexibly determine the need of non-anchor carrier and the allocation of contention free and contention based preambles so that the success ratio of random access can be improved while the allocated resource can also be effectively utilized.

This paper is organized as follows. Section 2 describes the related works of NB-IoT and its random access. The proposed non-anchor carrier and preamble allocation algorithm are provided in Section 3. In Section 4, the performance, which includes successful ratio and resource utilization, of the proposed algorithm is illustrated through exhaustive simulations with discussion. Finally, we conclude our works and propose future research in the last section.

\section{Related Works}

Unlike traditional Zadoff-Chu (ZC) sequence, the preamble of NB-IoT are constituted by four symbol groups and each group consists of one cyclic prefix (CP) and five symbols [4]. And the anchor carrier is determined by referring to the LTE DC subcarrier with fix interval. The anchor carrier provides 64 preambles for uplink random access. In release 13, only the anchor-carrier can be adopted for paging and random access, and it cannot support the high access attempts issued by huge number of IoT devices. In order to relax the congestion of ran- 
dom access, 3 GPP release 14 allows to allocate up to 15 non-anchor carriers for random access in addition to the original anchor carrier. Thus the release 13 UE can only use the anchor carrier for random access and the release 14 UE can use either anchor carrier or non-anchor carrier as shown in Figure 1.

However, if more non-anchor carriers are allocated for random access, it will decrease the radio resource to be used for data transmission. It is noted that, generally, the data to be transmitted by the IoT device is quite small when comparing to the traditional services. In [5], the small data transmission (SDT) scheme was proposed to allow data being piggybacked by the message 3 of the random access procedure as shown in Figure 2.

The eNB can arrange numbers preamble for contention free access and contention based access constrained by the total amount of allocated preambles. There is no collision for the device when it is assigned the contention free preamble. And for the devices without assigned contention free preamble will randomly choose one preamble from the contention based preambles. And the collision may occur. Once collision occurred, the random back-off time is applied to deal with the collision issue.

In [6], in addition to using access class barring (ACB), devices with different transmission behavior were differentiated and using different back-off times to

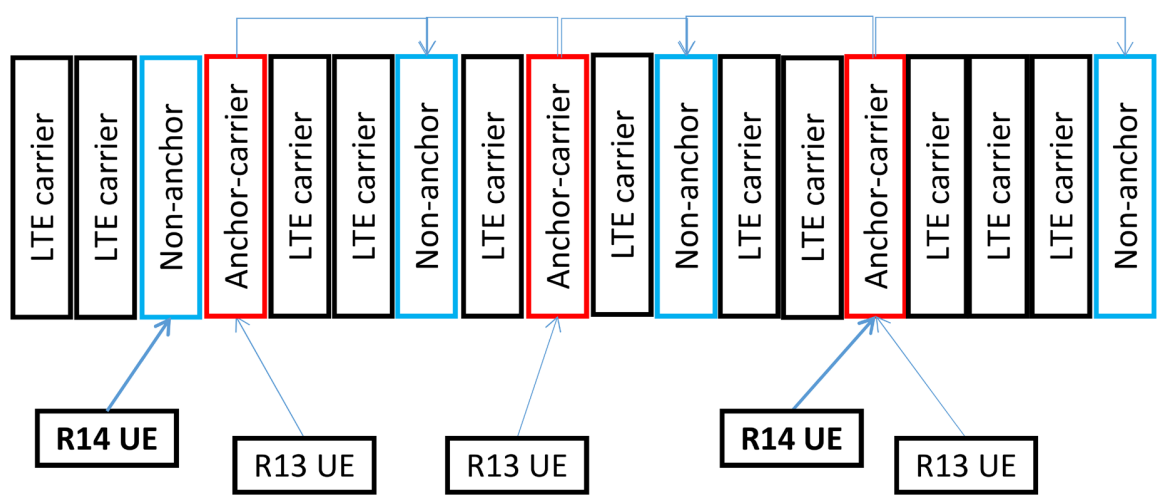

Figure 1. Anchor and non-anchor carriers for random access.

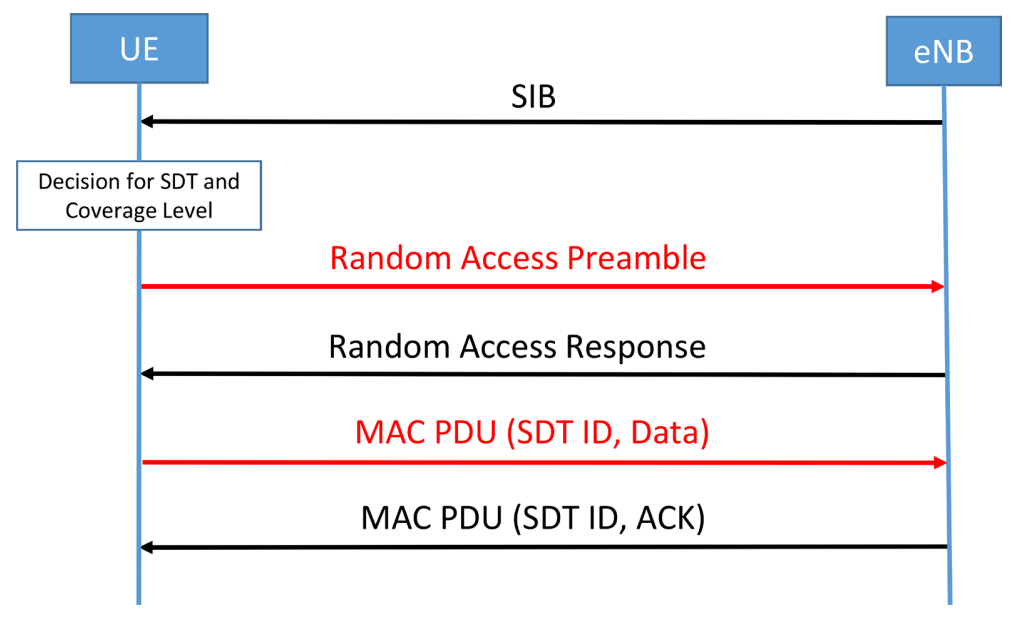

Figure 2. SDT in random access procedure. 
smooth the access congestion. The enhanced access algorithm based on clustering-reuse preamble allocation algorithm was proposed in [7]. The devices are divided into clusters by referring to the distance between the UE and the eNB, and the access intensity. The clusters with similar distance are allocated with complementary preamble sets and the same preamble set is allocated to clusters with long distance so that the preambles can be reused. However, there are several parameters, which include constraints and criteria during clustering, the numbers of preambles allocated to clusters, shall be properly determined so that the collision rate and system throughput can be effectively improved. Instead of uniform-distributed selection of contention preambles, the authors in [8] proposed the Poisson process-based random variable for preamble accessing to reduce the probability that multiple devices have equal chance to choose the identical contention preamble. Hence the numbers of preambles and access attempts and behavior are the critical parameters that affect the random access performance.

\section{Proposed System Model and CRF Algorithm}

In order to support flexible number of preambles, the non-anchor carriers can be allocated for random access in addition to the existing anchor carrier. The proposed system model focuses on the adaptive use of non-anchor carriers by referring to the congestion status of random access from end devices. The collision report flag (CRF) is designed by the feedback from the end device when it successfully sends the message 3 in the random access procedure. We adopt one of the reserved bits of the in Msg 3 together with data volume and power headroom report (DPR) MAC control element in Msg 3 together with a common control channel (CCCH) SDU as shown in Figure 3.

The CRF is set by each device to inform eNB about whether or not it suffered the collision before the success of random access. The setting procedure of CRF is illustrated in Figure 4. By using CRF, eNB can get the information of the ratio of devices that successfully contend the preamble without collision among all of the successful access devices. If the ratio is low, it means that most devices are hard to complete their access without any collision.

The proposed algorithm adaptively adjusts the number of used non-carriers by referring to the usage of preambles, the ratio of the number of successful decoded preambles to the used preambles detected by eNB, and the normalized CRF ratio. The normalized CRF ratio $R_{f}$ is calculated by the difference between the numbers of devices with CRF $=1$ and CRF $=0$ to the total number of successful transmission devices. The ratio is ranged between -1 to +1 . The negative value of $R_{f}$ means that the most devices have experienced at least one collision

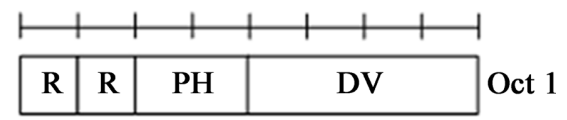

Figure 3. The reserved bit of DPR MAC control element in Msg 3. 
before successful transmission $(\mathrm{CRF}=1)$ is greater than that without any collision $(\mathrm{CRF}=0)$. The notations and descriptions of parameters and the thresholds used in the proposed algorithm, are provided in Table 1 and Figure 5, respectively.

There are three thresholds defined as follows. The preamble usage threshold is denoted as $T_{1}$, and the thresholds for the ratio of the successful decoded preambles to the number of all detected preambles when the preamble usage ratio is high and low are represented as $T_{2}$ and $T_{3}$, respectively. These three thresholds are applied in the proposed algorithm to determine the adjustment of non-anchor carriers. The proposed algorithm first examines the preamble usage ratio $R_{u}$, if it is less than $T_{1}$, then it checks the ratio of the successfully decoded preambles to see whether to reduce the number of non-anchor carrier or not. If the preamble usage ratio is high (i.e. $R_{u} \geq T_{1}$ ), then the proposed algorithm, in addition to comparing the ratio of successfully decoded preambles with $T_{2}$, the normalized CRF ratio will also be checked whether the allocated non-anchor carriers is still enough. The higher value of $R_{f}$ means that most end devices can successfully

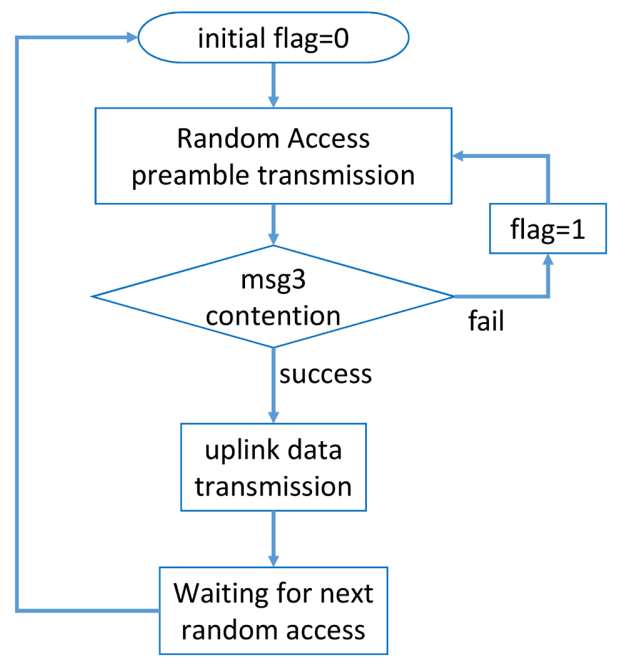

Figure 4. The setting procedure of collision report flag.

Table 1. Notations and definitions used in the proposed algorithm.

\begin{tabular}{cc}
\hline Notations & Descriptions \\
\hline$N_{d}$ & Number of preambles detected by eNB \\
$N_{t}$ & Number of total preambles allocated by eNB \\
$R_{u}$ & The usage of preamble $\left(P_{d} / P_{t}\right)$ \\
$N_{s}$ & Number of success UEs \\
$S_{f}$ & Sum of CRF flags \\
$R_{f}$ & Ratio of $S_{f}$ and $N_{s}\left(S_{f} / N_{s}\right)$ \\
$C$ & Total non-anchor carriers \\
$M_{1}$ & Maximum number of non-anchor carriers \\
$T_{1}, T_{2}, T_{3}$ & Thresholds used in the proposed algorithm
\end{tabular}


transmit the preamble without collision. Thus the normalized CRF ratio plays an important role to decide whether the number of allocated non-anchor carriers shall be decreased, increased, or without change. The non-anchor carrier will be either increased or decreased when $R_{f}$ is in the range $\{-1,-0.5\}$ or $\{0.5,1\}$, respectively. And it will be no change if $R_{f}$ is within the range between -0.5 and 0.5 . It is noted that the change of total number preambles is forty-eight for the increment or decrement of one non-anchor carrier. Therefore, the maximum number of increased non-anchor carrier is two under the constraint of $\mathrm{M}$. The procedure of the proposed CRF algorithm is provided in Figure 6.

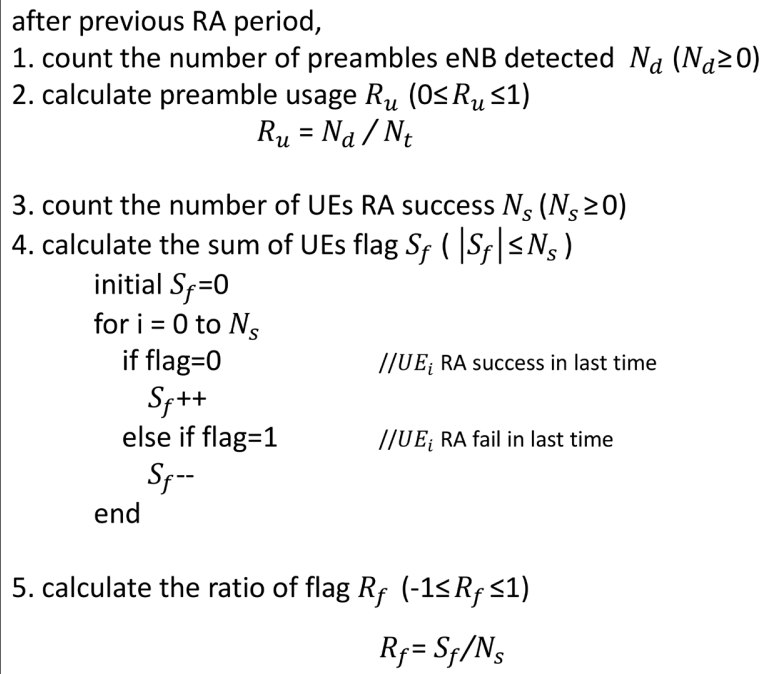$$
R_{f}=S_{f} / N_{s}
$$

Figure 5. The thresholds adopted in the proposed CRF algorithm.

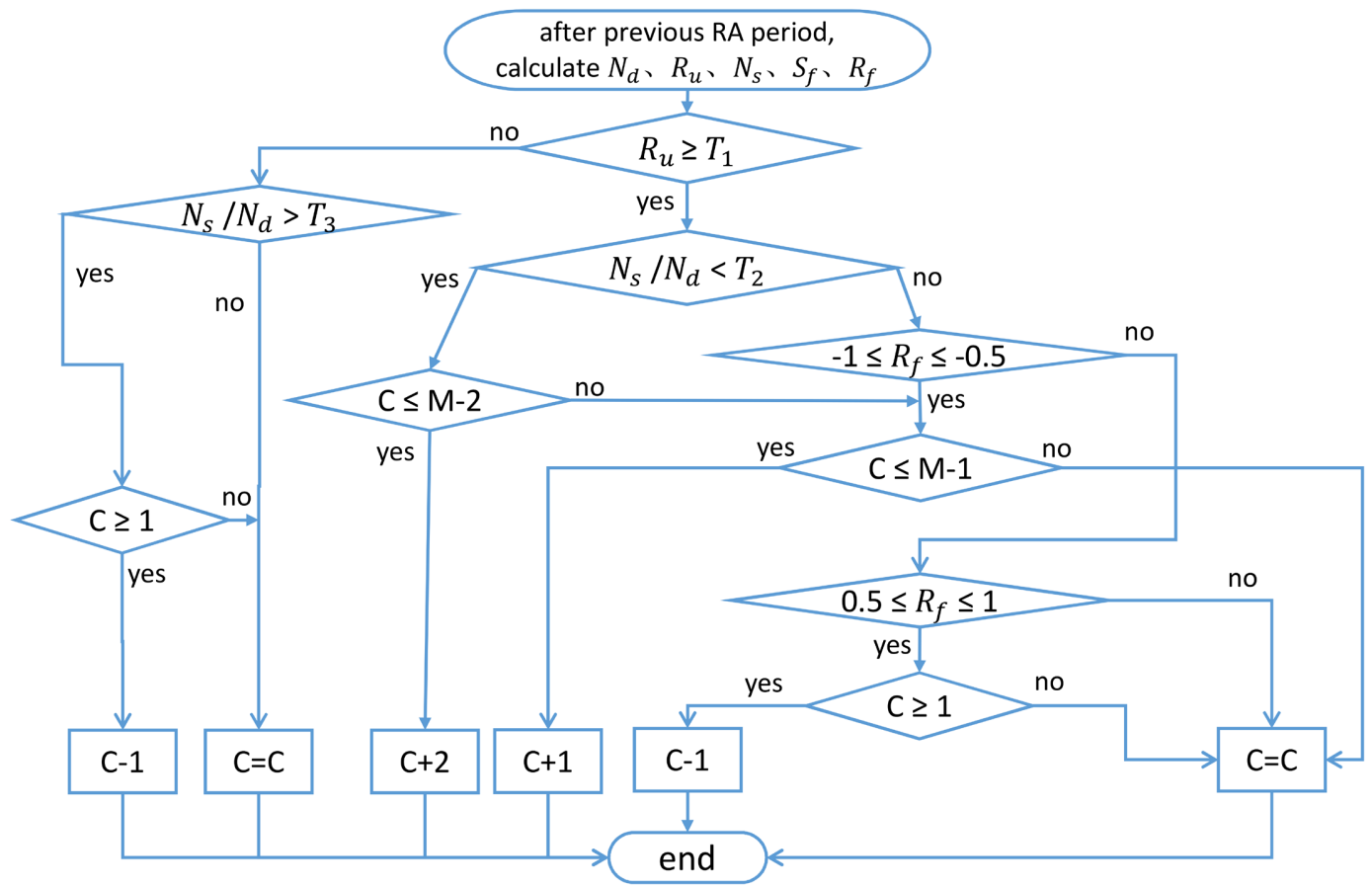

Figure 6. The procedure of the proposed CRF algorithm of non-anchor carrier adjustment. 
It is noted that there are three random access cycles required to get the CRF sent in message 3, therefore, the adjustment of non-carrier is performed for every three random access cycles as shown in Figure 7.

\section{Experimental Results}

The effectiveness of the proposed algorithm was conducted through simulations and compared to that of the scheme with fixed number of non-anchor carriers. The in band with $180 \mathrm{KHz}$ spectrum was adopted and the other parameters used during the simulations are listed in Table 2 as follows.

The simulation was performed for 10,000 times and 1280 random access period for each time. The simulation results were calculated from the average of the 10,000 times and only the results obtained during the period [20,9979] were counted in to prevent transient effect. Figure 8 shows an example of UE = 30,000 to illustrate the simulation results counted for average.

In order to find the suitable thresholds, $T_{1}, T_{2}$ and $T_{3}$, we performed simulations in advance to check their stability. The stability was defined as the frequency of the number of non-anchor carrier changed for the same number of UEs. The thresholds of $T_{1}, T_{2}$ and $T_{3}$ were set to be $0.5,0.6,0.9$, respectively, by referring to the results, in the simulations. The results of the proposed CRF algorithm were compared to the fixed number of non-anchor carriers. As the maximum number of non-anchor carriers is 15 , the numbers of non-anchor carriers of the compared fixed scheme were set to be 5, 10, and 15, respectively. Figure 9 shows the comparison of the success random access ratio of the proposed CRF

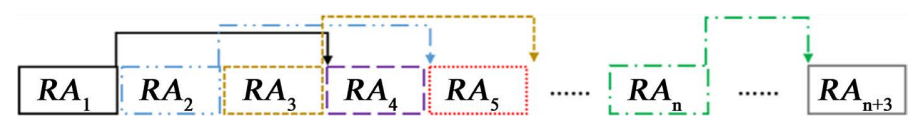

Figure 7. The adjustment cycle of non-anchor carrier.

Table 2. Simulation parameters.

\begin{tabular}{cc}
\hline Parameters & Values \\
Deployment & In Band \\
Number of UEs & $15,000-80,000(2500$ per point $)$ \\
Simulation time & $1280 * 10,000 \mathrm{~ms}$ \\
Traffic model & Poisson distribution \\
Mean of arrival & $0.003 \mathrm{Pkt} / \mathrm{s}, 0.045 \mathrm{Pkt} / \mathrm{s}$ \\
RA period & $1280 \mathrm{~ms}$ \\
Threshold $T_{1}, T_{2}, T_{3}$ & $50 \%, 60 \%, 90 \%$ \\
Number of anchor carriers & 1 \\
M & 12,15 \\
Number of preambles per carrier & 48 \\
Maximum retransmission times of UE (retx) & 5,10 \\
\hline
\end{tabular}




\section{Ex: UE $=30000$}

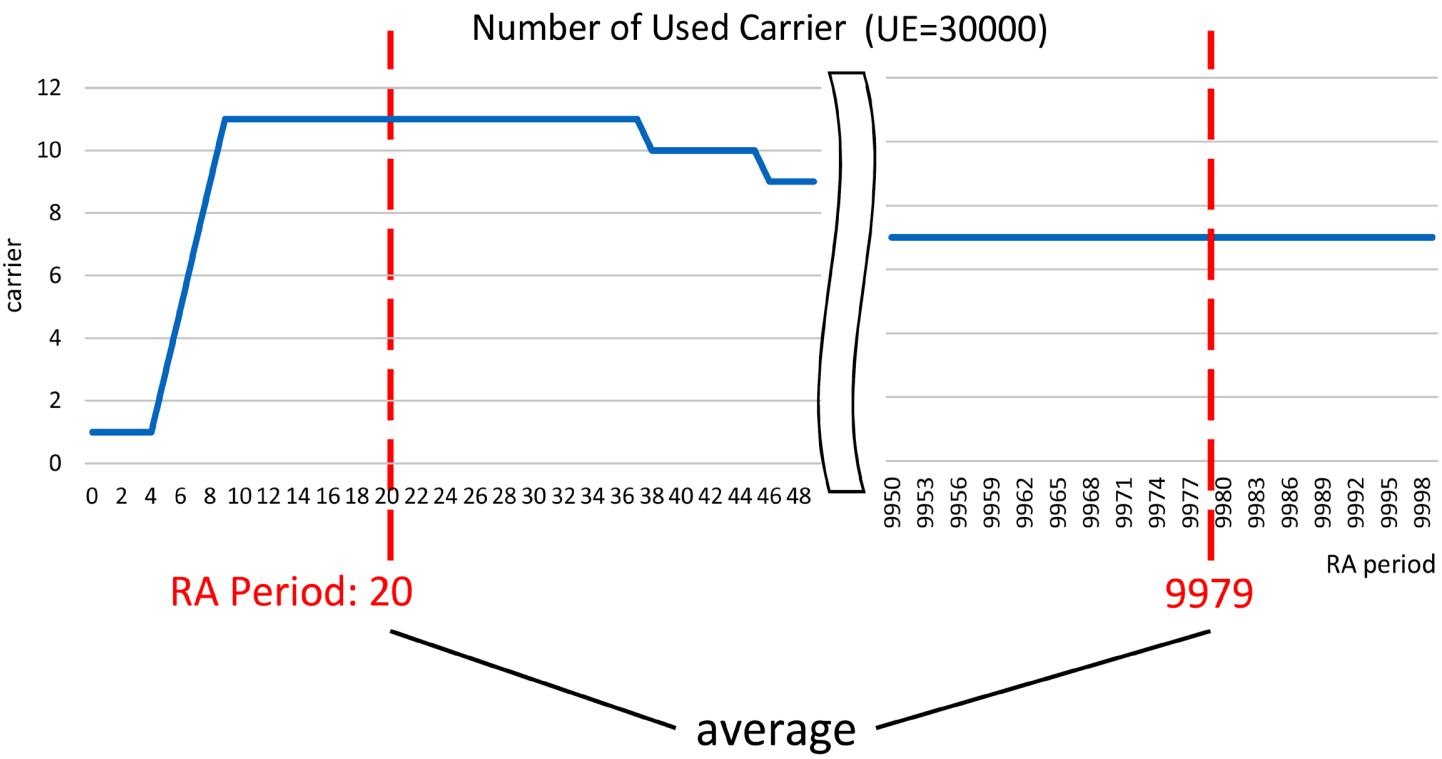

Figure 8. Simulation results counted for average period.

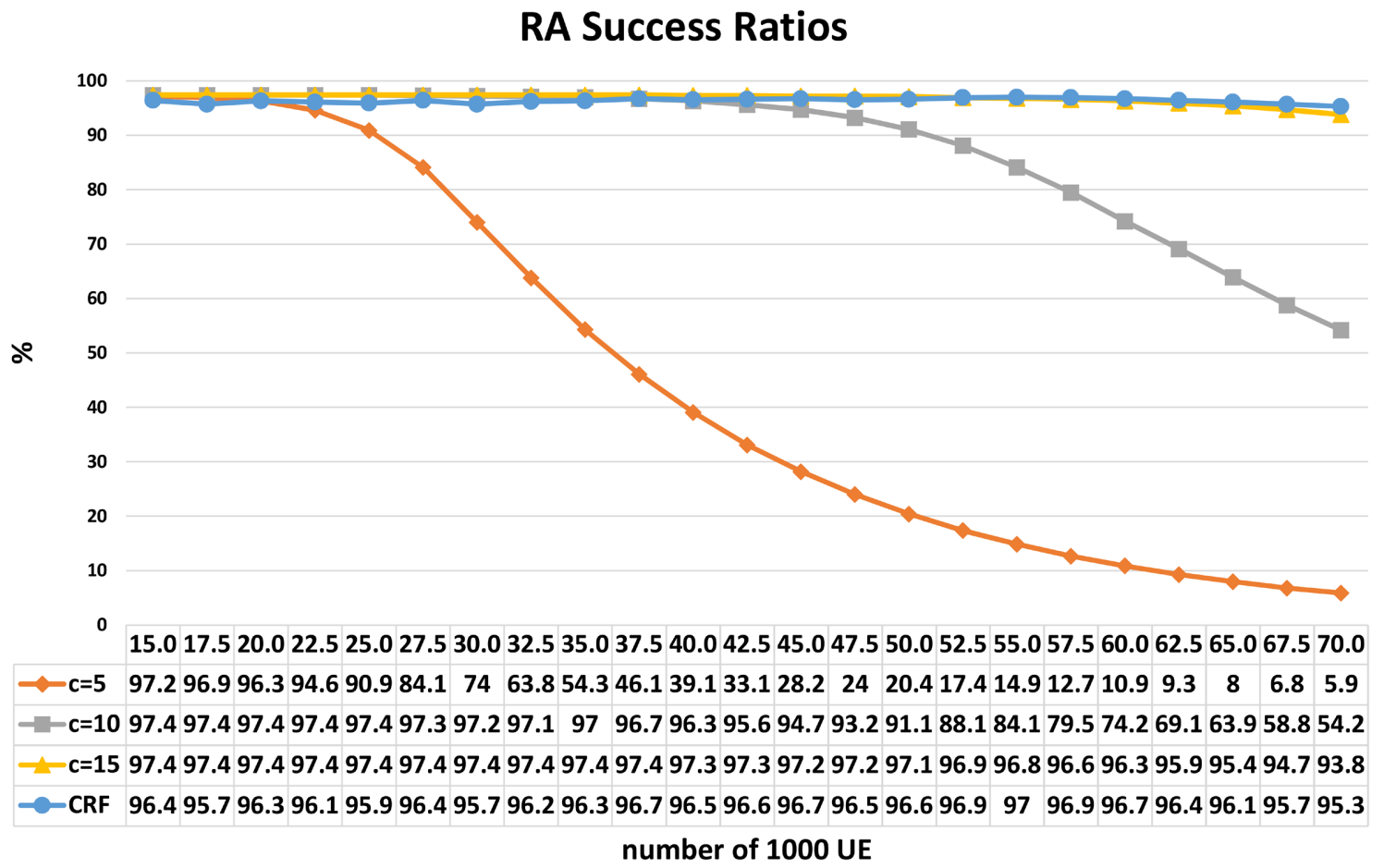

Figure 9. Comparisons of RA success ratios v.s. number of UEs.

algorithm and the fixed schemes. The results show that the success ratios start to decrease dramatically when the numbers of UE exceed 25,000 and 47,500 for the numbers of the non-anchor carriers are fixed to be 5 and 10, respectively. And the proposed algorithm performs almost the same RA success ratio to the fixed 15 non-anchor scheme in either small number of UEs or large number of UEs. 


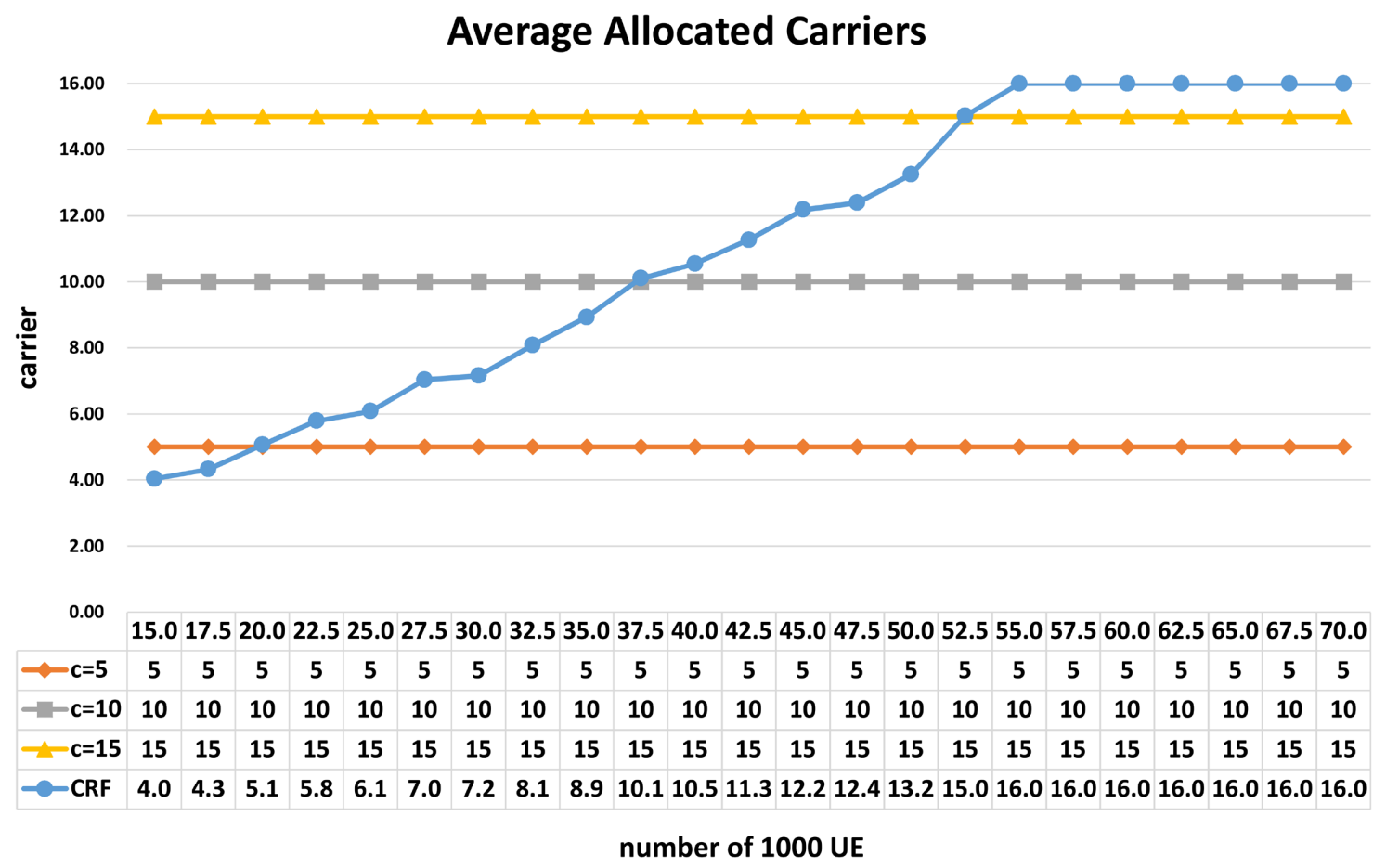

Figure 10. Comparisons of numbers of carriers allocated v.s. number of UEs.

Figure 10 compares the numbers of non-anchor carriers allocated for the proposed CRF algorithm and the fixed schemes. It is noted that the actual number of carriers used of the fixed schemes shall be 6,11 , and 16 , respectively, because the anchor-carrier is default to be used for random access. The result illustrates that the proposed scheme can dynamically allocate the number of non-anchor carriers as the number of UE increases accordingly.

Figure 11 provides the preamble utilization of the proposed scheme and the compared schemes. The utilization is defined as the number of preambles that are adopted by UE(s) regardless whether the collision occurred or not. Hence it is not suggested to have higher preamble utilization because the high preamble utilization may result in high collision ratio. It shows that the proposed algorithm has the stable preamble utilization. The utilization of the proposed algorithm maintains within $30 \% \sim 40 \%$. The utilization of the fixed 5 non-anchor scheme is about $60 \%$ when the number of UE is 30,000 . And its collision ratio starts to increase, which results in higher retransmission time as shown in Figure 12.

\section{Conclusions}

This paper proposed the dynamic non-anchor carrier allocation algorithm by referring to the feedback CRF by using one reserved bit of the data volume and power headroom report MAC control element in Msg 3. eNB can get this bit for each successful random access from end devices. This bit provides whether the end device has ever experienced collision before its successful transmission. And $\mathrm{eNB}$, in addition to the CRF, considers the preamble utilization, and the ratio of 


\section{The Utilizations of Allocated Preambles}

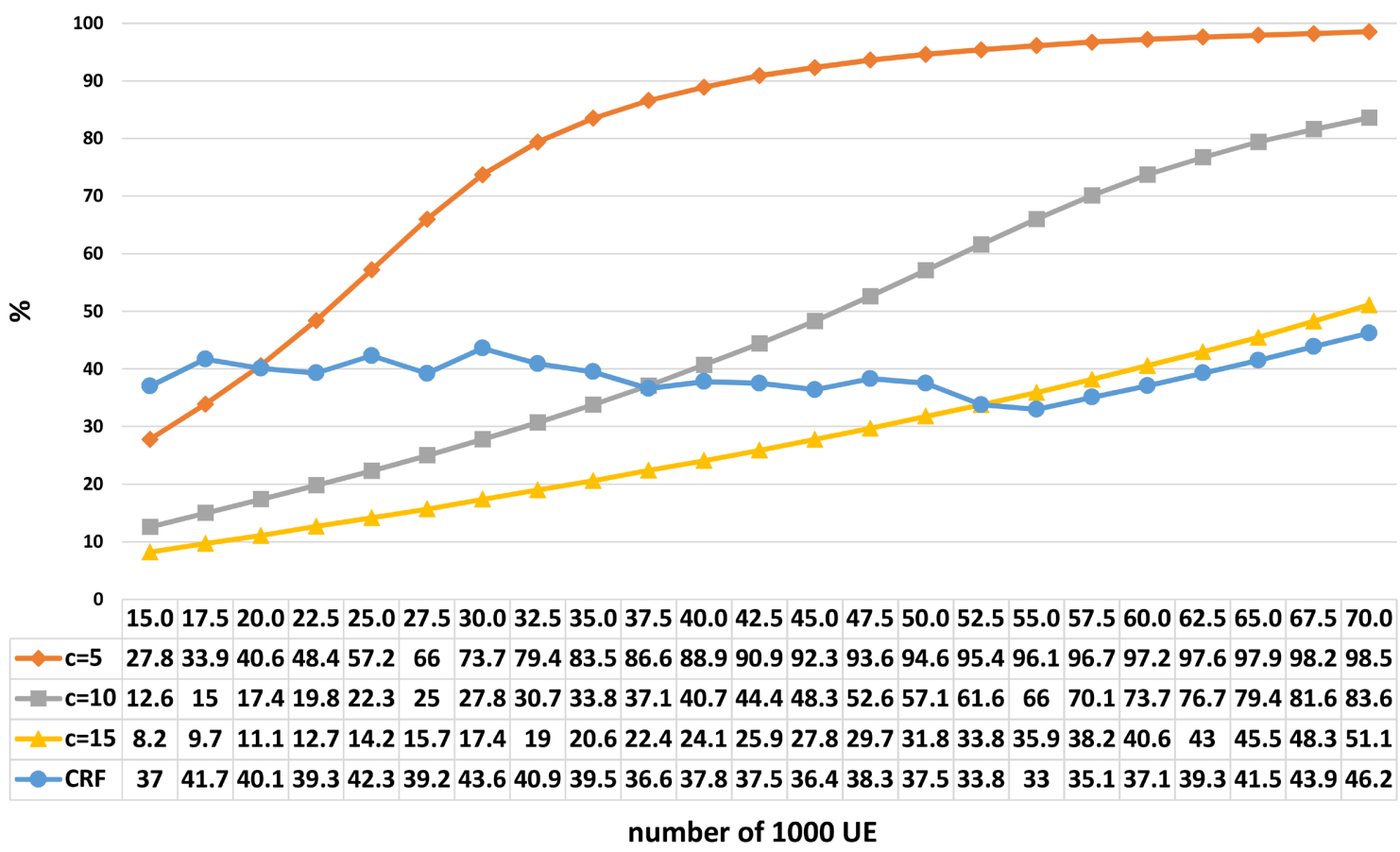

Figure 11. Comparisons of preamble utilizations.

\section{UE Average retx}

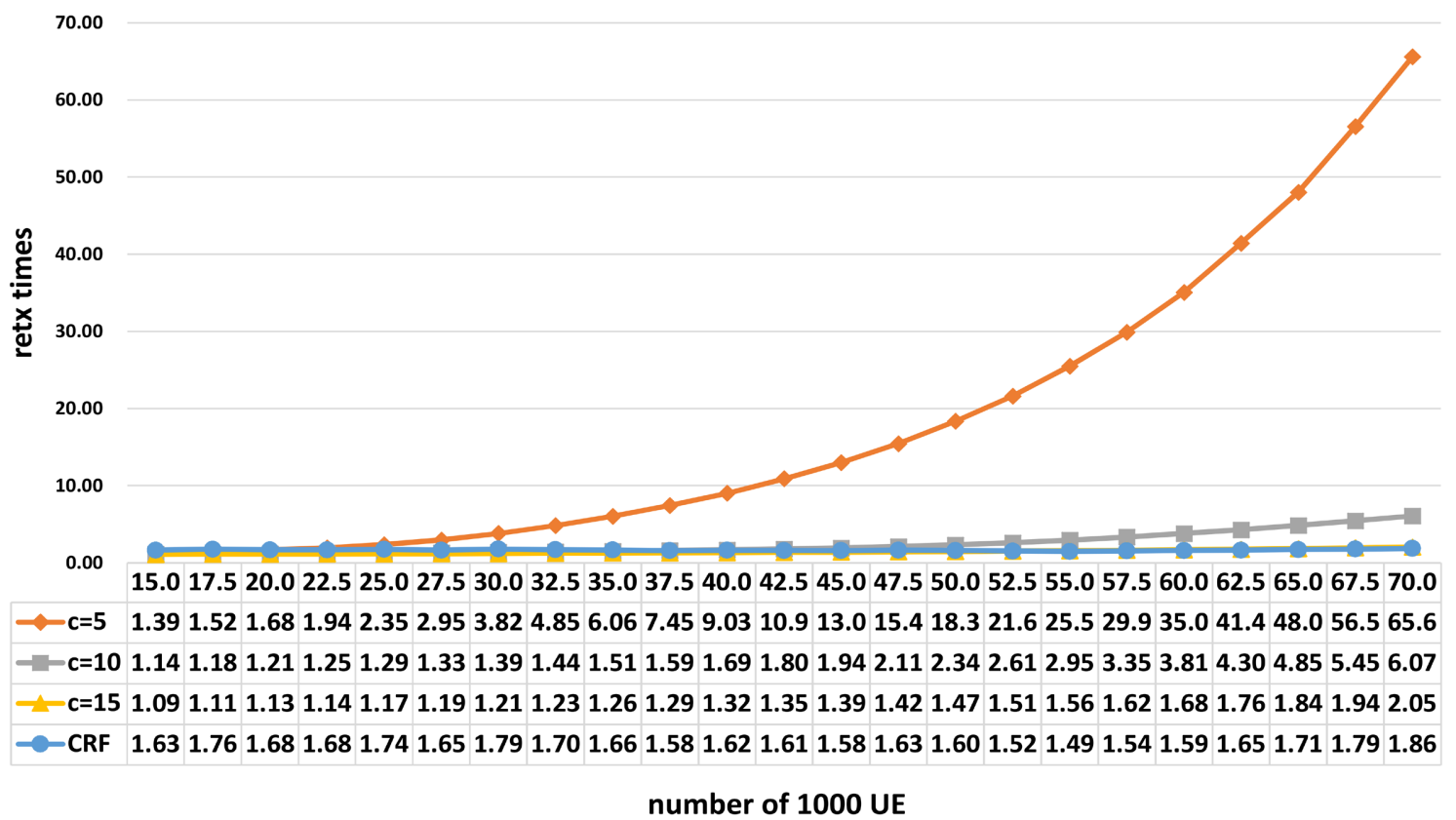

Figure 12. The average retransmission times per RA.

successful transmitted preamble, to determine the allocation of the number of non-anchor carriers The simulation results demonstrate that the proposed CRF algorithm performs almost the same successful RA ratio when comparing to the scheme with all non-anchor carriers fix-allocated. However, the proposed algo- 
rithm consumes much less non-anchor carriers when the number of end devices is small. For the low to medium traffic load, the proposed scheme can save 5 - 10 nun-anchor carriers when comparing to the fixed scheme.

The preamble utilization of the proposed algorithm is maintained around $40 \%$ and it may be possible to be further improved. Because we noted that the successful RA ratios of the fixed 5 and 10 non-anchor carrier schemes can achieve almost the same ratio as the proposed scheme when the numbers of UEs are below 25,000 and 47,500, respectively, and their preamble utilization is above $50 \%$. The proposed algorithm may be able to further improve radio resource allocation for RA. The using of machine learning is one of the possible approaches toward this objective and this is one of our ongoing research directions.

\section{Acknowledgements}

This work was supported in part by the Ministry of Science and Technology (MOST) (grant number: 109-2221-E-008-052-MY2), Taiwan.

\section{Conflicts of Interest}

The authors declare no conflicts of interest regarding the publication of this paper.

\section{References}

[1] Wang, Y.-P.E., Lin, X.Q., Adhikary, A., Grovlen, A., Sui, Y.T., Blankenship, Y., Bergman, J. and Razaghi, H. S. (2017) A Primer on 3GPP Narrowband Internet of Things. IEEE Communications Magazine, 55, 117-123. https://doi.org/10.1109/MCOM.2017.1600510CM

[2] 3GPP TR 21.914 (2018) Release 14 Description; Summary of Rel-14 Work Items. V14.0.0, May 2018.

[3] Narrowband Internet of Things Whitepaper (2019) https://cdn.rohde-schwarz.com/pws/dl downloads/dl application/application note s/1ma266/1MA266 0e NB IoT.pdf

[4] Lin, X.Q., Adhikary, A. and Wang, Y.-P.E. (2016) Random Access Preamble Design and Detection for 3GPP Narrowband IoT Systems. IEEE Wireless Communications Letters, 5, 640-643. https://doi.org/10.1109/LWC.2016.2609914

[5] Oh, S.-M. and Shin, J.S. (2017) An Efficient Small Data Transmission Scheme in the 3GPP NB-IoT System. IEEE Communications Letters, 21, 660-663. https://doi.org/10.1109/LCOMM.2016.2632128

[6] Zhao, Y.F., Liu, K., et al. (2018) A Classification Back-Off Method for Capacity Optimization in NB-IOT Random Access. 2017 11 th IEEE International Conference on Anti-Counterfeiting, Security, and Identification (ASID), Xiamen, 27-29 October 2017, 104-108. https://doi.org/10.1109/ICASID.2017.8285753

[7] Wu, F., et al. (2019) An Enhanced Random Access Algorithm Based on the Clustering-Reuse Preamble Allocation in NB-IoT System. IEEE Access, 7, 183847-183859. https://doi.org/10.1109/ACCESS.2019.2960436

[8] Almagrabi, A.O., Ali, R., Alghazzawi, D., AlBarakati, A. and Khurshaid, T. (2021) A Poisson Process-Based Random Access Channel for 5G and Beyond Networks. Mathematics, 9, Article No. 508. https://doi.org/10.3390/math9050508 